\title{
Quantificação e Avaliação das Principais Espécies Florestais Licenciadas no Estado do Acre de 2005 a 2012
}

\author{
Flávia Alessandra Pinheiro da Rocha Cordeiro e Silva ${ }^{1}$, \\ Renato Cesar Gonçalves Robert ${ }^{1}$, Adriana da Silva Santos ${ }^{2}$, \\ Sabina Dessartre Mendonça ${ }^{1}$ \\ ${ }^{1}$ Departamento de Engenharia e Tecnologia Florestal, Universidade Federal do Paraná - UFPR, Curitiba/PR, Brasil \\ ${ }^{2}$ Instituto Federal do Paraná - IFPR, Curitiba/PR, Brasil
}

\begin{abstract}
RESUMO
O presente trabalho teve como objetivo avaliar, identificar e quantificar os 15 principais gêneros e espécies florestais exploradas legalmente no estado do Acre, na Amazônia brasileira, bem como analisar o número de autorizações emitidas para exploração de florestas manejadas, o volume de madeira e a área de manejo licenciada pelo Instituto de Meio Ambiente do Acre de janeiro de 2005 a agosto de 2012. Os dados coletados demonstraram que a área e o volume de madeira licenciados apresentaram um crescimento no período analisado, atingindo o ápice em $2010 \mathrm{e}$ 2011, respectivamente. Os 15 principais gêneros e espécies licenciados foram: cumaru-ferro (Dipteryx spp.), garapeira (Apuleia spp.), sumaúma (Ceiba spp.), açacu (Hura crepitans L.), matamatá (Eschweilera spp.), jatobá (Hymenaea spp.), tauari (Couratari spp.), manitê (Brosimun spp.), caucho (Castilla ulei Warb.), cedro (Cedrela spp.), guariúba (Clarisia racemosa Ruiz e Pav.), faveira (Parkia spp.), copaíba (Copaifera spp.), guaribeiro (Phyllocarpus riedelii Tul.) e abiurana (Pouteria spp.).
\end{abstract}

Palavras-chave: manejo florestal, Autorização de Exploração, Instituto de Meio Ambiente do Acre.

\section{Quantification and Assessment of the Main Forest Species Licensed for Exploitation in the State of Acre from 2005 to 2012}

\begin{abstract}
The present study aimed to assess, identify and quantify the fifteen main forest genus and species legally exploited in the state of Acre, Brazilian Amazon, as well as to analyze the number of authorizations issued to the exploitation of managed forests, the total wood volume, and total managed area licensed by the Environmental Institute of Acre (IMAC) from January 2005 to August 2012. Gathered data showed that the licensed area and volume had an increase during the analyzed period, reaching the highest numbers in 2010 and 2011, respectively. The fifteen main genus and species licensed were: Cumaru-ferro (Dipteryx spp.), Garapeira (Apuleia spp.), Sumaúma or Silk Floss tree (Ceiba spp.), Açacu or Sandbox tree (Hura crepitans L.), Matamatá (Eschweilera spp.), Jatoba (Hymenaea spp.), Tauari (Couratari spp.), Manitê (Brosimun spp.), Caucho (Castilla ulei Warb.), Cedro (Cedrela spp.), Guariúba (Clarisia racemosa Ruiz \& Pav.), Faveira (Parkia spp.), Copaiba (Copaifera spp.), Guaribeiro (Phyllocarpus riedelii Tul.) and Abiurana or Pouteria trees (Pouteria spp.).
\end{abstract}

Keywords: forest management, Authorization of Exploitation, Environmental Institute of Acre (IMAC). 


\section{INTRODUÇÃO}

Há muitos anos vem-se discutindo a melhor forma de conciliar o desenvolvimento econômico com a conservação dos recursos naturais (Azevedo, 2006). Por um lado, o setor florestal desempenha um papel fundamental no cenário socioeconômico brasileiro, ao contribuir com a produção de bens e serviços, agregação de valor aos produtos florestais e geração de empregos, tributos e renda (Santos, 2011). Entretanto, com a crescente preocupação com a preservação da natureza, cada vez mais tem se colocado o desafio de buscar o desenvolvimento econômico aliado à conservação dos recursos naturais.

O reconhecimento da inadequada ocupação da Amazônia e da incompatibilidade entre o modelo desenvolvimentista e a evolução sustentável dessa floresta tem levado o estado do Acre a adotar políticas públicas voltadas à manutenção da biodiversidade $\mathrm{e}$ ao desenvolvimento sustentável (Acre, 2012).

Nesse contexto, o manejo florestal de impacto reduzido é, segundo Lima (2010), a melhor forma de gestão florestal para garantia da sustentabilidade. Esse sistema de exploração desenvolveu-se em resposta às demandas sociais pela conservação da floresta $\mathrm{e}$ proteção ambiental, e utiliza as melhores técnicas de extração disponíveis, reduzindo os danos às florestas residuais e o desgaste do solo, minimizando assim os riscos de erosão e auxiliando na manutenção da diversidade biológica (Holmes et al., 2004). Dessa forma, é imprescindível que o planejamento da exploração madeireira seja feito de forma adequada, garantindo a manutenção da floresta para outros ciclos de corte e assegurando a compatibilidade social do uso florestal (Sabogal et al., 2006). No entanto, segundo esses autores, atualmente há falta de informação, qualificação e interesse no que se refere ao manejo florestal e à qualidade de suas operações.

Conforme Hummel (2001), apenas na década de 1990 foram estabelecidos critérios técnicos para apresentação de planos de manejo florestal. A realização do manejo florestal depende da aprovação de um documento técnico denominado Plano de Manejo Florestal Sustentável (PMFS). De acordo com a Resolução Estadual Conjunta CEMACT/CFE n. 003/2008, além do PMFS o detentor da área na qual será realizado o manejo florestal deve apresentar ao órgão competente o Plano Operacional
Anual (POA) da Unidade de Produção Anual (UPA) a ser explorada. Após a análise dos planos pelo órgão licenciador, é emitida a Licença de Operação (LO) e a Autorização de Exploração (AUTEX), que autoriza o início da exploração da UPA e especifica o volume máximo por espécie permitido para exploração, com validade de 24 meses (Acre, 2008).

De acordo com a Secretaria de Meio Ambiente do Acre (Acre, 2012), cerca de $96 \%$ da matéria-prima de origem florestal no estado é proveniente de áreas com planos de manejo florestais sustentáveis, sendo que no fim da década de 1990 essa porcentagem era de apenas 5\%. A exploração das florestas naturais está intimamente relacionada com o suprimento da demanda industrial madeireira no Acre (Lima, 2010) e o manejo florestal é uma das prioridades do governo acreano desde 1999 (Sabogal et al., 2006). Segundo Pereira et al. (2010), o consumo de toras no Acre em 2009 foi de $422.000 \mathrm{~m}^{3}$, o que gerou uma receita bruta de 91,4 milhões de dólares. Todavia, a participação do Acre na produção nacional de madeira ainda é pequena (Silva, 2002). O desenvolvimento de políticas públicas que promovam o manejo florestal de impacto reduzido e o estudo de técnicas que otimizem o processo do manejo são de grande importância para elevar a participação do estado acreano no cenário nacional (Ribeiro, 2008).

As estatísticas relacionadas ao setor florestal geralmente são poucas e inconsistentes, o que pode prejudicar um debate produtivo e transparente (Hummel, 2001).É o que ocorre no caso do Instituto de Meio Ambiente do Acre (IMAC), onde a sociedade desconhece as informações das principais espécies florestais licenciadas pelo referido órgão e suas volumetrias ao longo dos anos, o que pode dificultar o controle dessas espécies no longo prazo. A divulgação de informações e a melhoria da eficiência do sistema de controle são variáveis de grande importância no desenvolvimento do manejo florestal sustentável.

Assim, o presente trabalho teve como objetivo avaliar as principais espécies florestais exploradas no Acre de 2005 a 2012, bem como quantificar a volumetria de madeira, o número de AUTEX emitidas e área licenciada nesse período a partir dos planos de manejo florestal submetidos ao IMAC, visando contribuir com a melhora operacional do setor florestal acreano. 


\section{MATERIAL E MÉTODOS}

\section{1. Área de estudo}

A pesquisa foi realizada no Instituto de Meio Ambiente do Acre, sediado em Rio Branco, Acre, nos meses de julho e agosto de 2012. Por se tratar de um órgão estadual, o estudo abrange todo o estado do Acre, composto por 22 municípios, localizados entre as latitudes $7^{\circ} 7^{\prime} \mathrm{S}$ e $11^{\circ} 8^{\prime} \mathrm{S}$ e as longitudes $66^{\circ} 30^{\prime} \mathrm{W}$ e $74^{\circ} 0^{\prime} \mathrm{W}$. As cinco microrregiões e cinco importantes municípios e uso e cobertura da terra (floresta nativa amazônica e ocupação humana) estão representados na Figura 1.

\subsection{Coleta dos dados}

A coleta dos dados foi realizada com o apoio da Divisão de Manejo Florestal (DIMF) do IMAC, sendo que os principais dados de todas as Autorizações de Exploração (AUTEX) do período de 1/1/2005 a 8/8/2012 foram digitalizados para posterior análise.

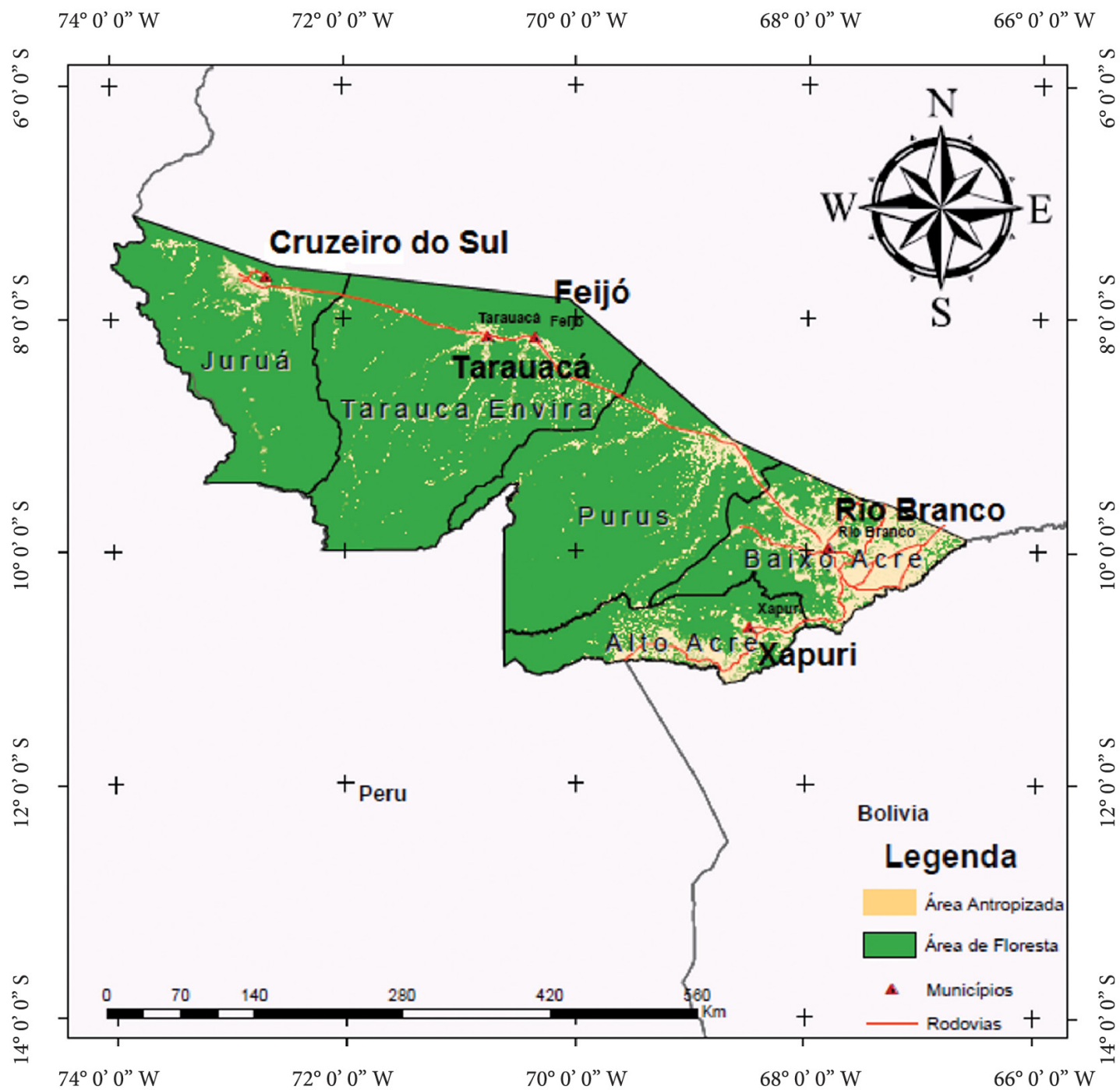

Figura 1. Mapa das microrregiões e municípios com uso e cobertura da terra (floresta nativa amazônica e ocupação humana) do estado do Acre em 2012.

Figure 1. Map displaying the state of Acre's municipalities and micro regions with land use and land cover (Amazon forest and human settlement) in 2012. 
Foram coletados os seguintes dados secundários: nome científico, nome vernacular, volume autorizado $\left(\mathrm{m}^{3}\right)$ de cada espécie, detentor do plano de manejo licenciado, volumetria total licenciada e área autorizada.

Devido ao grande número de espécies autorizadas anualmente, no presente estudo foram analisados os 15 principais gêneros e espécies, totalizando assim 65 espécies dentro dos 15 grupos analisados (Tabela 1). As espécies e gêneros analisados foram obtidos conforme informações contidas nas AUTEX. Para sumarização das informações, algumas espécies avaliadas foram agrupadas por gênero, visto que em alguns casos diferentes espécies do mesmo gênero possuíam o mesmo nome popular. Também fizeram parte desse agrupamento as espécies das quais apenas o gênero constava na autorização de exploração.

Todos os dados foram obtidos diretamente das Autorizações de Exploração. Porém, no ano de 2007 não foi encontrada a volumetria por espécie de 7 dos 36 planos de manejo licenciados nesse ano. No entanto, a volumetria e a área total puderam ser obtidas através da avaliação dos relatórios anuais emitidos pelo IMAC.

Para a realização dos objetivos propostos no presente trabalho, os dados foram sistematizados em tabelas anuais, contendo as informações anteriormente citadas. Para coleta e processamento dos dados foi utilizado um software de planilhas eletrônicas.

Tabela 1. Espécies e gêneros avaliados no presente estudo.

Table 1. Species and genus assessed in the study.

\begin{tabular}{|c|c|c|}
\hline Grupo & Espécies incluídas & Nome vulgar \\
\hline Dipteryx spp. & $\begin{array}{l}\text { Dipteryx micrantha Harms.; Dipteryx odorata (Aubl.) Willd. e Dip- } \\
\text { teryx polyphylla Huber }\end{array}$ & Cumaru-ferro \\
\hline Apuleia spp. & $\begin{array}{l}\text { Apuleia molaris Spruce ex Benth.; Apuleia leiocarpa (Vogel) J. F. } \\
\text { Macbr e Apuleia sp. }\end{array}$ & Garapeira \\
\hline Ceiba spp. & $\begin{array}{l}\text { Ceiba petandra (L.) Gaertn.; Ceiba samauma K. Schum.; Ceiba } \\
\text { sellowiana e Ceiba speciosa (A. St.-Hil.) Gibbs e Semir }\end{array}$ & $\begin{array}{l}\text { Sumaúma; } \\
\text { samaúma }\end{array}$ \\
\hline Hura crepitans L. & Hura crepitans L. & Açacu \\
\hline Eschweilera spp. & $\begin{array}{l}\text { Eschweilera compressa (Vell.) Miers; Eschweilera grandiflora (Aubl.) } \\
\text { Sandwith; Eschweilera ovata (Cambess.) Miers.; Eschweilera ama- } \\
\text { zônica; Eschweilera coriacea (DC) S. A. Mori; Eschweilera odora (Po- } \\
\text { epp. Ex O. Berg) Miers e Eschweilera juruensis e Eschweilera sp. }\end{array}$ & Matamatá \\
\hline Hymenaea spp. & $\begin{array}{l}\text { Hymenaea courbaril L.; Hymenaea oblongifolia Huber; Hymenaea capa- } \\
\text { nema Ducke; Hymenaea microphylla Barb. Rodr. e Hymenaea sp. }\end{array}$ & Jatobá \\
\hline Couratari spp. & $\begin{array}{l}\text { Couratari guianensis Aubl.; Couratari macrosperma A. C. Sm.; } \\
\text { Couratari tauari O.Berg; Couratari obongifolia Ducke e R. Knuth; } \\
\text { Couratari martiana (O. Berg) Miers e Couratari sp. }\end{array}$ & Tauari \\
\hline Brosimun spp. & $\begin{array}{l}\text { Brosimun uleanum; Brosimun lecointei Ducke; Brosimun parina- } \\
\text { rioides Ducke; Brosimun acutifolium Huber; Brosimun lactescens } \\
\text { (S. Moore) C. C. Berg; Brosimun utile (H. B. K.) Pittier; Brosimun } \\
\text { alicastrum Sw. e Brosimun sp. }\end{array}$ & Manitê \\
\hline Castilla ulei Warb. & Castilla ulei Warb. & Caucho \\
\hline Cedrela spp. & Cedrela fissilis Vell.; Cedrela odorata L. e Cedrela sp. & Cedro \\
\hline Clarisia racemosa Ruiz e Pav. & Clarisia racemosa Ruiz e Pav. & Guariúba \\
\hline Parkia spp. & $\begin{array}{l}\text { Parkia paraensis Ducke; Parkia pendula (Wild.) Benth. Ex Walp.; } \\
\text { Parkia gigantocarpa; Parkia multijuga Benth.; Parkia nitida Miq.e } \\
\text { Parkia sp. }\end{array}$ & Faveira \\
\hline Copaifera spp. & $\begin{array}{l}\text { Copaifera langsdorffi Desf.; Copaifera multijuga Hayne; Copaifera } \\
\text { guianensis Desf.; Copaifera duckei Dwyer e Copaifera sp. }\end{array}$ & Copaíba \\
\hline Phyllocarpus riedelii Tul. & Phyllocarpus riedelii Tul. & Guaribeiro \\
\hline Pouteria spp. & $\begin{array}{l}\text { Pouteria anomala (Pires) T. D. Penn.; Pouteria caimito (Ruiz e Pav.) } \\
\text { Radlk.; Pouteria guianensis Aubl.; Pouteria macrophylla (Lam.) } \\
\text { Eyma; Pouteria pachycarpa Pires; Pouteria bangii; Pouteria oblance- } \\
\text { olata Pires; Pouteria oppositifolia (Ducke) Baehni; Pouteria williamii } \\
\text { e Pouteria sp. }\end{array}$ & Abiurana \\
\hline
\end{tabular}




\section{RESULTADOS E DISCUSSÃO}

O número de Autorizações de Exploração expedidas pelo IMAC teve um aumento no período analisado, o que pode ser visto na Figura 2. Mediante essas informações, constatou-se que o maior número de AUTEX emitidas foi de 55, no ano de 2010. O ano de 2012 inclui as AUTEX expedidas até o mês de agosto, o que explica o baixo número nesse ano. Ainda assim pode-se afirmar que a partir de 2010 houve um decréscimo no número de Autorizações de Exploração emitidas, extrapolando os valores das AUTEX para os demais meses do ano de 2012

Segundo Oliveira \& Braz (1998), em 1998 a maior parte da madeira produzida no estado do Acre ainda era originária de áreas de conversão. Em levantamento de empresas florestais em Rio Branco em 1996, Silva (1996) constatou que apenas uma empresa utilizava madeira oriunda de planos de manejo florestal. Entre 2007 e 2010, houve um aumento do licenciamento ambiental no estado do Acre, que pode ser expresso pelo aumento do número de AUTEX expedidas. Segundo a Secretaria de Estado e Meio Ambiente do Acre (Acre, 2006), em 2006 cerca de 85\% do total da madeira autorizada para consumo era oriunda de planos de manejo, o que indica uma melhora na utilização racional dos recursos florestais.

Ao realizar um histórico do licenciamento ambiental no Acre de 2000 a 2009, Lima (2010) constatou que a regional que possui maior área, volumetria de madeira e número de planos de manejo licenciados ao longo desse período é a regional Baixo Acre. Tal fato pode ter ocorrido em função dessa regional possuir um maior desenvolvimento, tanto de infraestrutura quanto urbanístico, facilitando assim o escoamento da produção.

A Figura 3 demonstra que volume anual de madeira autorizada sofreu um acréscimo com o passar do tempo, com uma ligeira redução no ano de 2008. O volume de madeira em 2005 foi de $284.800 \mathrm{~m}^{3}$, enquanto em 2011 esse volume chegou a $769.353 \mathrm{~m}^{3}$, o que representou um aumento de quase $270 \%$. Tal fato se opõe aos outros estados da Amazônia Legal, onde houve redução na extração madeireira na última década (Pereira et al., 2010). Segundo esses autores, o Acre foi o único estado que apresentou um aumento na extração de madeira em tora, bem como aumento na receita

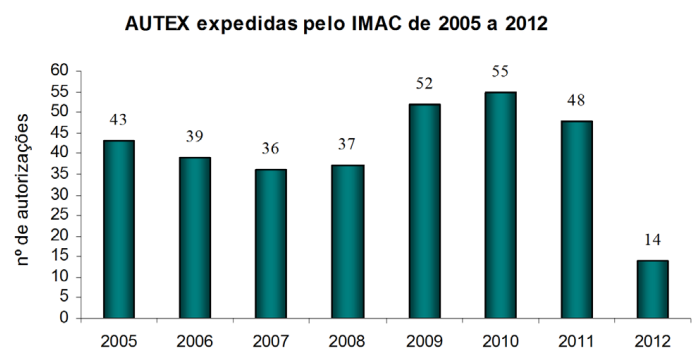

Figura 2. Número de Autorizações de Exploração no período de 2005 a 2012.

Figure 2. Number of Authorizations of Exploitation from 2005 to 2012.

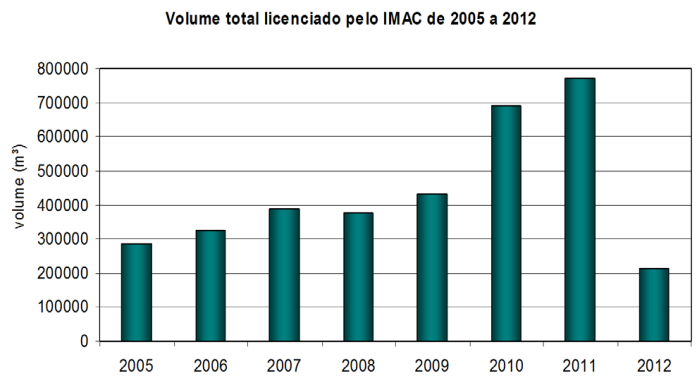

Figura 3. Volume total de madeira autorizado pelo IMAC de 2005 a 2012.

Figure 3. Total wood volume authorized by IMAC from 2005 to 2012

bruta do setor madeireiro entre 1998 e 2009, passando de R 21 milhões para R 182 milhões (SFB, 2010). Esses aumentos podem ter ocorrido em função do aumento do nível de industrialização do setor e das políticas públicas de estímulo ao setor florestal, que ampliaram a origem de madeira oriunda de planos de manejo nos últimos anos (Acre, 2006).

O volume licenciado em 2012 foi de apenas $214.183 \mathrm{~m}^{3}$, mas tal volume representa o total licenciado até o mês de agosto, deixando de abranger os demais meses do ano. É importante salientar que o volume total licenciado pelo Instituto de Meio Ambiente do Acre não é necessariamente o volume efetivamente explorado, e que o volume extraído deve obrigatoriamente estar contido no relatório pós-exploratório entregue pelo detentor ao IMAC.

Os resultados referentes ao volume anual e total dos 15 principais grupos de espécies licenciadas de 2005 a agosto de 2012 podem ser observados na Tabela 2. As principais espécies e gêneros explorados legalmente 
no estado do Acre durante o período analisado, segundo seu nome vernacular e em ordem decrescente, foram: cumaru-ferro (Dipteryx spp.), garapeira (Apuleia spp.), sumaúma (Ceiba spp.), açacu (Hura crepitans L.), matamatá (Eschweilera spp.), jatobá (Hymenaea spp.), tauari (Couratari spp.), manitê (Brosimun spp.), caucho (Castilla ulei Warb.), cedro (Cedrela spp.), guariúba (Clarisia racemosa Ruiz e Pav.), faveira (Parkia spp.), copaíba (Copaifera spp.), guaribeiro (Phyllocarpus riedelii Tul.) e abiurana (Pouteria spp.).

As Autorizações de Exploração do ano de 2007 não localizadas no setor de arquivo técnico tornaram-se um problema no momento do levantamento dos dados. Somente os dados de volume total estavam disponíveis, não existindo, assim, o detalhamento da exploração por espécies nesse ano. Esse fato pode explicar a queda do volume autorizado dos principais grupos de espécies no presente ano, visto que os resultados do volume por espécie abrangeram $67 \%$ do total de planos licenciados.

Os 15 principais gêneros e 65 espécies licenciadas correspondem a $62,26 \%$ do volume total autorizado de 2005 a 2012, apesar de representarem apenas 5,59\% do número total de espécies identificadas apenas pelo nome vernacular licenciadas nesse mesmo período. Tal fato corrobora o que afirmou Silva (2002), que expressou que, no Acre e em outros estados da Amazônia brasileira, ocorre uma intensa exploração de poucas espécies, o que pode colocar em risco a manutenção de sua população ao longo do tempo. O Instituto Brasileiro de Meio Ambiente e Recursos Naturais Renováveis (IBAMA, 2010) também afirmou, em análise das espécies nativas transportadas pelo sistema DOF (Documento de Origem Florestal) no Brasil, que no período de 2007 a 2009 apenas 25 espécies correspondiam a $50 \%$ de todo o volume transportado, indicando que o mercado continua restrito a um número de espécies relativamente pequeno.

Robert et al. (2012), ao avaliarem o abastecimento de madeira serrada em Florianópolis, observaram que os estabelecimentos comerciais madeireiros dessa região costumam adquirir um baixo número de espécies florestais tropicais e que o consumidor final não demanda outras espécies que vêm sendo exploradas em planos de manejo florestal sustentável. Segundo esses autores, 47\% dos estabelecimentos analisados trabalham com apenas duas espécies, o que reforça a falta de diversidades de espécies comercializadas na região. Contudo, a inserção de novas espécies de madeira no mercado pode ser considerada um grande desafio, visto que, apesar de haver uma diversidade de espécies na Amazônia brasileira com potencial madeireiro, poucas são conhecidas pelo mercado consumidor, que geralmente busca por espécies mais populares e tradicionais.

Tabela 2. Volume dos 15 principais grupos de espécies licenciadas pelo IMAC de 2005 a 2012.

Table 2. Volume of the 15 main groups of species licensed by IMAC from 2005 to 2012.

\begin{tabular}{|c|c|c|c|c|c|c|c|c|c|c|c|}
\hline \multirow{2}{*}{\multicolumn{2}{|c|}{ NOME CIENTÍFICO }} & \multicolumn{10}{|c|}{ VOLUME NO PERÍODO ANALISADO $\left(\mathrm{m}^{3}\right)$} \\
\hline & & \multirow{2}{*}{$\begin{array}{l}2005 \\
33.506\end{array}$} & \multirow{2}{*}{$\begin{array}{l}2006 \\
41.693\end{array}$} & \multirow{2}{*}{$\begin{array}{c}2007 \\
16.698\end{array}$} & \multirow{2}{*}{$\begin{array}{l}2008 \\
35.737\end{array}$} & \multirow{2}{*}{$\begin{array}{c}2009 \\
50.827\end{array}$} & \multirow{2}{*}{$\begin{array}{l}2010 \\
61.839\end{array}$} & \multirow{2}{*}{$\begin{array}{l}2011 \\
64.533\end{array}$} & \multirow{2}{*}{$\begin{array}{l}2012 \\
21.541\end{array}$} & \multirow{2}{*}{$\begin{array}{c}\text { TOTAL } \\
326.373\end{array}$} & \multirow{2}{*}{$\begin{array}{r}\text { MÉDIA } \\
40.797\end{array}$} \\
\hline 1 & Dipteryx spp. & & & & & & & & & & \\
\hline 2 & Apuleia spp. & 29.229 & 30.096 & 17.786 & 32.022 & 38.739 & 53.532 & 67.113 & 19.554 & 288.071 & 36.009 \\
\hline 3 & Ceiba spp. & 25.887 & 32.260 & 25.404 & 23.434 & 41.999 & 60.000 & 59.279 & 15.722 & 283.984 & 35.498 \\
\hline 4 & Hura crepitans & 1.835 & 6.298 & 8.092 & 16.286 & 23.512 & 38.927 & 32.920 & 13.170 & 141.039 & 17.630 \\
\hline 5 & Eschweilera spp. & 2.901 & 1.772 & 12.789 & 18.059 & 21.989 & 36.649 & 39.839 & 6.942 & 140.939 & 17.617 \\
\hline 6 & Hymenaea spp. & 11.859 & 15.790 & 8.317 & 17.415 & 16.145 & 27.091 & 28.193 & 8.101 & 132.911 & 16.614 \\
\hline 7 & Couratari spp. & 13.553 & 9.887 & 13.881 & 17.441 & 17.047 & 19.642 & 28.677 & 6.432 & 126.560 & 15.820 \\
\hline 8 & Brosimun spp. & 6.400 & 8.750 & 11.683 & 16.571 & 16.409 & 25.227 & 29.972 & 8.202 & 123.212 & 15.402 \\
\hline 9 & Castilla ulei & 8.255 & 9.757 & 3.555 & 11.455 & 14.908 & 31.156 & 29.861 & 7.674 & 116.621 & 14.578 \\
\hline 10 & Cedrela spp. & 9.191 & 12.074 & 6.323 & 9.251 & 10.136 & 17.287 & 21.462 & 7.248 & 92.973 & 11.622 \\
\hline 11 & Clarisia racemosa & 4.523 & 3.413 & 9.769 & 11.117 & 10.132 & 19.945 & 22.013 & 5.134 & 86.045 & 10.756 \\
\hline 12 & Parkia spp. & 2.605 & 3.309 & 5.102 & 12.839 & 10.481 & 19.319 & 23.161 & 6.962 & 83.778 & 10.472 \\
\hline 13 & Copaifera spp. & 5.897 & 6.337 & 4.314 & 11.036 & 10.291 & 16.986 & 17.355 & 5.773 & 77.988 & 9.748 \\
\hline 14 & Phyllocarpus riedelii & 1.095 & 3.394 & 5.112 & 10.449 & 11.265 & 18.953 & 21.565 & 3.824 & 75.657 & 9.457 \\
\hline \multirow[t]{2}{*}{15} & Pouteria spp. & 3.491 & 2.309 & 8.732 & 9.244 & 10.411 & 18.099 & 17.099 & 2.592 & 71.979 & 8.997 \\
\hline & $\Sigma$ & 160.227 & 187.137 & 157.557 & 252.355 & 304.290 & 464.653 & 503.042 & 138.870 & 2.168 .130 & \\
\hline
\end{tabular}


Tabela 3. Volume total autorizado $\left(\mathrm{m}^{3} \mathrm{e}^{3} \cdot \mathrm{ha}^{-1}\right)$ e área de manejo florestal autorizada pelo IMAC de 2005 a 2012. Table 3. Total volume authorized (cu. m. and cu. m. per hectare) and forest management area authorized by IMAC from 2005 to 2012.

\begin{tabular}{cccc} 
Ano & $\begin{array}{c}\text { Área autorizada } \\
(\mathbf{h a})\end{array}$ & Volume autorizado $\left.\mathbf{( m}^{\mathbf{3}}\right)$ & $\begin{array}{c}\text { Volume autorizado } \\
\left(\mathbf{m}^{\mathbf{3}} \mathbf{h} \mathbf{a}^{-\mathbf{1}}\right)\end{array}$ \\
\hline 2005 & 17.899 & 284.800 & 15,9 \\
2006 & 27.206 & 326.008 & 12,0 \\
\hline 2007 & 22.403 & 387.878 & 17,3 \\
2008 & 26.571 & 378.032 & 14,2 \\
\hline 2009 & 28.683 & 431.613 & 15,0 \\
\hline 2010 & 42.945 & 690.257 & 16,1 \\
\hline 2011 & 42.802 & 769.353 & 18,0 \\
\hline 2012 & 12.151 & 214.183 & 17,6 \\
\hline MÉDIA & $\mathbf{2 7 . 5 8 3}$ & $\mathbf{4 3 5 . 2 6 6}$ & $\mathbf{1 5 , 8}$ \\
\hline$\Sigma$ & $\mathbf{2 2 0 . 6 6 0}$ & $\mathbf{3 . 4 8 2 . 1 2 6}$ & \\
\hline
\end{tabular}

A Fundação de Tecnologia do Estado do Acre (FUNTAC, 1990) observou que, em 1990, a exploração madeireira no estado concentrava-se em sete espécies, que representaram $79,21 \%$ do total processado naquele ano. As espécies mais utilizadas nesse período, em ordem decrescente, foram: mogno (Swietenia macrophylla L.), cerejeira (Amburana acreana Ducke), cedro (Cedrela spp.), sumaúma (Ceiba spp.), jatobá (Hymenaea spp.), angelim (Hymenolobium spp.) e copaíba (Copaifera spp.).

Segundo o trabalho de Silva (2002), entre 2000 e 2001, as dez espécies mais licenciadas pelo IBAMA, em ordem decrescente, foram: cumaru-ferro (Dipteryx spp.), sumaúma, garapeira, cedro, cerejeira, jatobá, copaíba, angelim, açacu (Hura spp.), mururé (Brosimun spp.) e abiu (Micropholis spp. e Pouteria spp). Tais espécies representaram $78,3 \%$ do volume total autorizado.

Ao analisar os dados de Silva (2002) e da FUNTAC (1990) percebe-se que grande parte do número de espécies exploradas em 1990 e em 2000/2001 continuam sendo exploradas no período de 2005 a 2012, o que pode ocasionar a diminuição da população ao longo do tempo e aumentar o risco de extinção local de algumas espécies. Em 1990 já ocorria uma exploração intensa de poucas espécies, uma vez que apenas sete espécies representaram 79,21\% do volume total explorado (FUNTAC, 1990). Mais de duas décadas depois, tal situação continua sendo um problema no manejo florestal, o que indica a necessidade de adoção de políticas que incentivem a introdução de novas espécies no mercado para não colocar em risco os estoques atuais.
A área de manejo florestal autorizada pelo Instituto de Meio Ambiente do Acre e o volume autorizado por hectare entre os anos de 2005 e 2012 podem ser visualizados na Tabela 3 . As áreas licenciadas somam um total de 220.660 ha, ocorrendo um aumento entre 2005 e 2011, com ápice em 2010 e 2011. Isso demonstra a proporcionalidade entre área e volume licenciado, uma vez que os períodos que apresentaram os maiores volumes de madeira autorizados foram os que tiveram as maiores áreas licenciadas, corroborando o estudo realizado por Lima (2010). Outro aspecto que deve ser considerado é aumento de políticas públicas estaduais para o fortalecimento do setor florestal, em especial o incentivo ao manejo florestal comunitário.

Em 2007, houve uma redução de quase 18\% da área autorizada para manejo, embora o volume total autorizado tenha aumentado nesse ano. Assim, conforme pode ser visto na Tabela 3, ocorreu o licenciamento de maior volume por hectare no presente ano. $\mathrm{O}$ volume licenciado de 2005 a 2012 foi de $15,8 \mathrm{~m}^{3} \mathrm{ha}^{-1} \mathrm{em}$ média, o que pode ser considerado um valor baixo, uma vez que a intensidade de corte máxima permitida pela legislação para a maioria dos planos de manejo submetidos ao licenciamento é de $30 \mathrm{~m}^{3} \mathrm{ha}^{-1}$.

\section{CONCLUSÕES}

Com base no desenvolvimento do presente estudo e nos resultados e discussões realizadas, pode-se concluir que: 
- O número de AUTEX e a área de manejo florestal autorizadas pelo Instituto de Meio Ambiente do Acre aumentaram no período de 2007 a 2010 e o volume licenciado teve um acréscimo durante o período de 2005 a 2011, com uma ligeira queda em 2008;

- Os 15 principais gêneros que incluem 65 espécies licenciadas no período avaliado foram responsáveis por pouco menos de $2 / 3$ do volume total autorizado, demonstrando uma intensa exploração de poucos gêneros.

\section{STATUS DA SUBMISSÃO}

Recebido: 26 dez., 2012

Aceito: 17 abr., 2015

\section{AUTOR(ES) PARA CORRESPONDÊNCIA}

\section{Flávia Alessandra Pinheiro da Rocha Cordeiro e Silva}

Departamento de Engenharia e Tecnologia

Florestal, Universidade Federal do Paraná -

UFPR, Rua Lothário Meissner, 900, Campus III, CEP 80210-170, Curitiba, PR, Brasil

e-mail: lamec.ufpr@gmail.com

\section{REFERÊNCIAS}

Acre. Secretaria de Estado e Meio Ambiente - SEMA. Zoneamento Ecológico-Econômico do Acre Fase II: Documento Síntese - Escala 1:250.000. Rio Branco; 2006.

Acre. Procuradoria-Geral do Estado do Acre. Resolução CEMACT/CFE $n^{\circ}$ 003, de 12 de agosto de 2008. Licenciamento, monitoramento e a fiscalização das áreas objeto de manejo florestal no Estado do Acre. Diário Oficial do Estado do Acre, Rio Branco, AC (2008 ago.). [citado 2012 Ago 22]. Disponível em: http://www.imc.ac.gov.br/wps/wcm/ connect/b1e91c0040b690558e73efed6d581285/Normas_ Ambientais_do_Estado_do_Acre_2ed.pdf?MOD=AJPERES.

Acre. Secretaria de Estado de Meio Ambiente - SEMA. $O$ Acre: no caminho da sustentabilidade. Rio Branco; 2012.

Azevedo CP. Dinâmica de florestas submetidas a manejo na Amazônia ocidental: experimentação e simulação [tese]. Curitiba: Setor de Ciências Agrárias, Universidade Federal do Paraná; 2006.

Fundação de Tecnologia do Estado do Acre - FUNTAC. Inventário Florestal e diagnóstico da regeneração da Floresta Estadual do Antimari. Rio Branco; 1990.
Holmes TP, Blate GM, Zweede JC, Pereira R Jr, Barreto P, Boltz F. Custos e benefícios financeiros da exploração de impacto reduzido em comparação com a exploração florestal convencional na Amazônia Oriental. 2. ed. Belém: Fundação Floresta Tropical; 2004.

Hummel AC. Normas de Acesso ao Recurso Florestal na Amazônia Brasileira: o caso do manejo florestal madeireiro [dissertação]. Manaus: Instituto Nacional de Pesquisas da Amazônia; 2001.

Instituto Brasileiro do Meio Ambiente e dos Recursos Naturais Renováveis - IBAMA. DOF: Informação estratégica para gestão florestal no Brasil: período 2007-2009. 2. ed. Brasília; 2010.

Lima VM. Uma década de licenciamento ambiental para planos de manejo florestal no Acre: de 2000 a 2009 [monografia]. Rio Branco: Universidade Federal do Acre; 2010.

Oliveira MVN, Braz EM. Manejo florestal em regime de rendimento sustentado aplicado à floresta do campo experimental da Embrapa-CPAF/AC. Rio Branco: EmbrapaCPAF/AC; 1998.

Pereira D, Santos D, Vedoveto M, Guimarães J, Veríssimo A. Fatos florestais da Amazônia 2010. Belém: IMAZON; 2010.

Ribeiro EP. Gestão de impacto ambiental com Plano de Manejo Florestal Sustentável empresarial: estudo do modelo digital de exploração florestal [dissertação]. Rio Branco: Universidade Federal do Acre; 2008.

Robert RCG, Santos AS, Santos LD, Fantini AC. Caracterização do abastecimento de madeira serrada comercializada no município de Florianópolis - SC. Floresta 2012; 42(1): 85-94. http://dx.doi.org/10.5380/rf.v42i1.26304.

Sabogal C, Lentini M, Pokorny B, Silva JNM, Zweede J, Veríssimo A et al. Manejo florestal empresarial na Amazônia Brasileira: restrições e oportunidades. Belém: Center for International Forestry Research; 2006.

Santos AS. Panorama do comércio de madeira serrada e perspectivas para o comércio de madeira certificada na Microrregião Geográfica de Florianópolis [dissertação]. Florianópolis: Universidade Federal de Santa Catarina; 2011.

Serviço Florestal Brasileiro - SFB. Instituto do Homem e Meio Ambiente da Amazônia - IMAZON. A atividade madeireira na Amazônia brasileira: produção, receita e mercados. Belém; 2010.

Silva RF. Diagnóstico das espécies mais utilizadas e alternativas para utilização nas serrarias localizadas na regional do Baixo Acre [dissertação]. Rio Branco: Universidade Federal do Acre; 2002.

Silva ZAGPG. Subsídios técnicos para a elaboração de uma estratégia de marketing para bens madeireiros produzidos no Estado do Acre. Rio Branco: FUNTAC; 1996. 